\title{
Letter About: Risk Factors for Mortality in Patients with COVID-19 in New York City
}

J Gen Intern Med 36(3):811-2

DOI: $10.1007 / \mathrm{s} 11606-020-06369-\mathrm{x}$

(C) Society of General Internal Medicine 2021

$\mathrm{D}^{\mathrm{e}}$ ear Editor,

We read with interest the study by Mikami et al. ${ }^{1}$ about the association of hydroxychloroquine (HCQ) with mortality in 3708 patients hospitalized with COVID-19. The authors reported adjusted HRs from a Cox regression model with and without propensity score adjustment, respectively: 0.53 , 95\% CI (0.41-0.68) and 0.53, 95\%CI (0.41-0.67). They concluded that treatment with HCQ was associated with reduced mortality. We appreciate that the authors appropriately tempered their interpretation of the results. Nonetheless, we are concerned that many readers may still overinterpret the impressive hazard ratios.

Moreover, we believe that the validity of the findings is weakened due to survivor bias, treatment selection bias, and reporting bias.

First, authors did not account for survivor bias in their analysis. Looking at their survival curves suggests that most deaths in the non-HCQ group occurred within 10 days of admission. $\mathrm{We}^{2}$ and other investigators ${ }^{3}$ have illustrated that survivor bias, which occurs because patients who live longer are more likely to receive treatment than those who die early, could change associations from benefit to harm. In a re- analysis of British hospital data from the Influenza Clinical Information Network study of 1391 patients with confirmed pandemic influenza A/H1N1 2009, authors observed that time bias can make Oseltamivir appear more effective (time-dependent bias), useless (competing risk bias), or even harmful (length bias). ${ }^{3}$

Second, surprisingly authors did not report on ICU care or ventilatory support in their cohort. Data from two large US cohorts ${ }^{4,5}$ during the same months of the pandemic reported that many patients died outside the ICU without ventilatory support (Table 1). Including these patients in the analysis would certainly affect the validity of the results due to confounding by indication. No statistical method can account for this treatment selection bias.

Finally, authors did not report on cardiac toxicity of HCQ in their cohort. Our group ${ }^{6}$ has recently conducted a metaanalysis on HCQ-induced cardiac toxicity in COVID-19 patients. We found that treatment with HCQ was associated with a clinically significant increased risk of QTc prolongation and discontinuation of drug due to QT prolongation. In addition, HCQ was associated with a clinically significant risk of torsades de pointes ventricular tachycardia (TdP) or monomorphic VT or cardiac arrest of 3 per 1000 (95\%CI 0.0-21).

We call for investigators to comply with reporting guidelines and for more vigilance in interpreting findings from observational studies especially when they show results contradicting those of randomized trials.
In response to:

Mikami, T., Miyashita, H., Yamada, T. et al. Risk Factors for Mortality in Patients with COVID-19 in New York City. J GEN INTERN MED (2020). https://doi.org/10.1007/s11606-020-05983-Z

Received July 11, 2020

Accepted November 25, 2020

Published online January 11, 2021 
Table 1 Comparison of Three Large COVID-19 Cohorts in New York and Michigan, USA-March to April 2020

\begin{tabular}{|c|c|c|c|c|}
\hline Arshad et al. & \multicolumn{4}{|c|}{ The Henry Ford Health System (6 hospitals) in Southeast Michigan: March 10, 2020 to May 2, 2020} \\
\hline Variable & HCQ group $(n=1985)$ & Non-HCQ $(n=556)$ & $N=2541$ & Comment \\
\hline ICU admission & $26.9 \%$ & $14.6 \%$ & $24.2 \%$ & $166 / 615(27 \%)$ of those who died did not \\
\hline Ventilatory support & $20.2 \%$ & $8.6 \%$ & $17.7 \%$ & receive mechanical ventilation \\
\hline Mortality & $16.1 \%$ & $25.4 \%$ & $18.1 \%$ & \\
\hline Richardson et al. & \multicolumn{4}{|c|}{$\begin{array}{l}\text { The Northwell Health System (12 hospitals) in New York City, Long Island, and Westchester County, New York: March 1, } \\
2020 \text {, and April 4, } 2020\end{array}$} \\
\hline Variable & HCQ group & Non-HCQ & $N=5700$ & $271 / 553(49 \%)$ and $262 / 553(47.4 \%)$ of those who \\
\hline ICU admission & NA & NA & $6.54 \%$ & died did not receive mechanical ventilation \\
\hline Ventilatory support & NA & NA & $14.2 \%$ & or ICU care, respectively \\
\hline Mortality & NA & NA & $9.7 \%$ & \\
\hline Mikami et al. & \multicolumn{4}{|c|}{ The Mount Sinai Health System (8 hospitals) in New York City: March 13 and April 17, 2020} \\
\hline Variable & HCQ group $(n=2073)$ & Non-HCQ $(n=742)$ & $N=2815$ & NR/NR (NR\%) of those who died did \\
\hline ICU admission & NR & NR & NR & not receive mechanical ventilation \\
\hline Ventilatory support & NR & NR & NR & \\
\hline Mortality & $27.5 \%$ & $31.1 \%$ & $28.5 \%$ & \\
\hline
\end{tabular}

HCQ, hydroxychloroquine. NA, not applicable (did not examine HCQ). NR, not reported

Imad M. Tleyjeh, MD, MSc, FACP, FIDSA ${ }^{1,2,3,4}$

Tarek Kashour, $M B C h B^{5}$

${ }^{1}$ Infectious Diseases Section, Department of Medical Specialties, King Fahad Medical City,

Riyadh, Saudi Arabia

${ }^{2}$ Division of Infectious Diseases, Mayo Clinic College of Medicine and Science,

Rochester, MN, USA

${ }^{3}$ Division of Epidemiology, Mayo Clinic College of Medicine and Science,

Rochester, MN, USA

${ }^{4}$ College of Medicine, Alfaisal University,

Riyadh, Saudi Arabia

${ }^{5}$ Department of Cardiac Sciences, King Fahad Cardiac Center, King Saud University Medical City, King Saud University,

Riyadh, Saudi Arabia

Corresponding Author: Imad M. Tleyjeh, MD, MSc, FACP, FIDSA; Infectious Diseases Section, Department of Medical Specialties, King Fahad Medical City, Riyadh, Saudi Arabia (e-mail: Tleyjeh.imad@mayo.edu).

\section{Compliance with Ethical Standards:}

Conflict of Interest: Authors report no conflict of interest.

\section{REFERENCES}

1. Mikami, T., Miyashita, H., Yamada, T. et al. Risk Factors for Mortality in Patients with COVID-19 in New York City. J Gen Intern Med. 2020. https://doi.org/10.1007/s11606-020-05983-Z.

2. Tleyjeh IM, Ghomrawi HM, Steckelberg JM, et al. Conclusion about the association between valve surgery and mortality in an infective endocarditis cohort changed after adjusting for survivor bias. J Clin Epidemiol. 2010;63(2):130-135. https://doi.org/10.1016/j.jclinepi.2008.06.022.

3. Wolkewitz $\mathbf{M}$, Schumacher $\mathbf{M}$. Survival biases lead to flawed conclusions in observational treatment studies of influenza patients. J Clin Epidemiol. 2017;84:121-129. https://doi.org/10.1016/j.jclinepi.2017.01.008.

4. Arshad S, Nauriyal V, et al. Treatment with Hydroxychloroquine, Azithromycin, and Combination in Patients Hospitalized with COVID-19. Int J Infect Dis. O(0). https://doi.org/10.1016/j.ijid.2020.06.099.

5. Richardson S, Hirsch JS, Narasimhan M, et al. Presenting Characteristics, Comorbidities, and Outcomes Among 5700 Patients Hospitalized With COVID-19 in the New York City Area. JAMA. 2020;323(20):20522059. https://doi.org/10.1001/jama.2020.6775.

6. Tleyjeh IM, Kashour Z, AlDosary $\mathbf{O}$, et al. The Cardiac Toxicity of Chloroquine or Hydroxychloroquine in COVID-19 Patients: A Systematic Review and Meta-regression Analysis. Mayo Clin Proc Innov Qual Outcomes. 2020. https://doi.org/10.1016/j.mayocpiqo.2020.10.005.

Publisher's Note: Springer Nature remains neutral with regard to jurisdictional claims in published maps and institutional affiliations. 